\title{
Influência do tipo de parto no resultado do tratamento fisioterapêutico na incontinência urinária
}

\author{
Influence of the type of delivery on the outcome of urinary incontinence \\ physical therapy treatment
}

\author{
Mara Regina Knorst ${ }^{1}$, Flávia Elize Lisot ${ }^{2}$, Larissa Lovatel Zollner ${ }^{2}$, \\ Maria Cristina Barbosa ${ }^{2}$, Thais de Lima Resende ${ }^{3}$
}

\begin{abstract}
RESUMO
Objetivos: Analisar a influência do tipo de parto na força muscular do assoalho pélvico (FMAP) de mulheres com incontinência urinária (IU), antes e depois de intervenção fisioterapêutica e determinar a relação entre os tipos de IU e tipos de parto.

Materiais e Métodos: Neste ensaio clínico, de braço único e não controlado foram incluídas 46 mulheres (35 a 78 anos) com diagnóstico médico de IU (de esforço - IUE, de urgência - IUU ou mista - IUM), que não realizaram cirurgia ginecológica. As voluntárias foram avaliadas e submetidas a tratamento conservador, constituído de exercícios perineais e eletroestimulação transvaginal.

Resultados: A média de idade foi de 53,6 $\pm 10,5$ anos. Metade das voluntárias apresentou IUM, 39,1\% apresentaram IUE e 10,9\% IUU. Após o tratamento, as mulheres que realizaram parto normal apresentaram aumento significativo da FMAP, tanto mensurada pelo perineômetro ( $p=0,031$; teste $t$ student para amostras pareadas), quanto pelo teste bidigital ( $p \leq 0,05$; teste de Wilcoxon), enquanto que as mulheres que realizaram parto cesárea ou os dois tipos de parto não apresentaram ganho significativo. A relação entre os tipos de parto e tipos de IU não apresentou diferença significativa. Mais de $80 \%$ das mulheres que realizaram parto normal ou cesárea se mostraram continentes ou satisfeitas após o tratamento.

Conclusão: As voluntárias que realizaram parto normal apresentaram o maior ganho da FMAP. Não obstante, os tipos de partos não influenciaram os resultados da intervenção fisioterapêutica relativos à continência urinária, uma vez que a maioria das mulheres tratadas relatou estar continente ou satisfeita com o tratamento.
\end{abstract}

Palavras-chave: incontinência urinária; parto; mulheres; saúde da mulher; gestação.

\section{ABSTRACT}

Objectives: To analyze the influence of the type of delivery on the pelvic floor muscle strength (PFMS) of women with urinary incontinence (UI), before and after physical therapy intervention and the relationship between the different types of $U$ I and type of delivery.

Materials and Methods: In this nonrandomized, uncontrolled clinical trial, 46 women (35 to 78 years) with a medical diagnosis of UI (stress - SUI, mixed - MUI, urgency - UUI), who did not undergo gynecological surgery were included. The voluntaries were evaluated (perineometry and bidigital test) and undertook a conservative treatment consisting of pelvic floor exercise and transvaginal electrical stimulation.

Results: The mean age was $53.6 \pm 10.5$ years. Half the voluntaries had MUI, $39.1 \%$ had SUI and $10.9 \%$ UUI. After treatment, the women who had had normal delivery presented a significant increase in the PFMS, both measured by the perineometer ( $p=0.031$; paired Student's $T$ test) and by the bidigital test ( $p \leq 0.05$; Wilcoxon's Test), while the women who had cesarean section or both types of delivery did not present a significant gain. The relationship between the type of delivery and types of UI did not present a significant difference. Over $80 \%$ of the women that had normal delivery or cesarean section were continent or satisfied after the treatment.

Conclusion: The voluntaries that had normal delivery presented the greatest gains in the PFMS. Notwithstanding, the type of delivery did not influence the results of the physical therapy intervention regarding urinary continence, since the majority of the women treated reported being continent or satisfied with the treatment.

Keywords: urinary incontinence; parturition; women; women's health; pregnancy.

\footnotetext{
${ }^{1}$ Fisioterapeuta. Doutora em Gerontologia Biomédica (PUCRS). Professora do Curso de Fisioterapia da Pontifícia Universidade Católica do Rio Grande do Sul (FAENFI-PUCRS).

${ }^{2}$ Fisioterapeuta graduada pela PUCRS.

${ }^{3}$ Fisioterapeuta. Doutora em Ciências da Saúde (PUCRS). Professora do Curso de Fisioterapia (FAENFI-PUCRS).
} 


\section{INTRODUÇÃo}

Segundo a Sociedade Internacional de Continência, a incontinência urinária é definida como qualquer perda involuntária de urina ${ }^{1,2}$. Na maioria dos estudos, a prevalência de incontinência urinária (IU) varia entre 10 a $40 \%$, apresentando um aumento gradual com a idade, com um pico de prevalência em torno dos 50 anos de idade, seguido por um ligeiro declínio ou estabilização até cerca de 70 anos, quando a prevalência se mostra em constante aumento ${ }^{3}$.

Há três tipos de IU: a de esforço (IUE), a de urgência (IUU) e a mista (IUM). A IUE ocorre quando há perda involuntária de urina ao esforço, ao exercício e ao espirrar ou tossir, a IUU ocorre se há vontade súbita e repentina de urinar que não pode ser postergada e IUM quando há associação de ambas as situações citadas acima, podendo também ser decorrente de aspectos emocionais ${ }^{4}$.

Durante a gestação a queixa de IU é constante e decorre da elevação da pressão intra-abdominal, pelo aumento do volume uterino que se transmite a bexiga e pela mudança do posicionamento da porção proximal da uretra $^{1}$. Segundo Scarpa et al. $^{5} 57,5 \%$ das pacientes apresentaram IUE durante a gestação e 56,5\% dessas tornaram-se assintomáticas até três anos após o parto. Tanto a gestação quanto o parto são momentos de grandes mudanças anatômicas e fisiológicas para o organismo materno, podendo resultar em alteração na função do trato urinário, manifestada por $I^{6}$.

O parto normal pode determinar graus de lesão muscular, nervosa e de tecido conjuntivo resultando na incontinência urinária e fecal, e ainda pode propiciar prolapsos de órgãos pélvicos ao longo da vida da mulher. As alterações anatômicas e funcionais do assoalho pélvico, que ocorrem durante o período expulsivo do parto podem regredir gradualmente, com restauro parcial ou completo do mecanismo da continência urinária ${ }^{5}$.

Além do parto, a episiotomia é motivo de várias controvérsias com relação à sua real eficácia na proteção das lesões perineais ${ }^{7}$. Oliveira e Miquilini ${ }^{7}$ observaram que a episiotomia médio-lateral direita foi a mais utilizada $(92,0 \%)$, comparando com a episiotomia mediana. $\mathrm{Na}$ episiotomia médio-lateral direita, além da pele e mucosa, normalmente são seccionados os músculos transverso superficial do períneo e bulbocavernoso.

Os tratamentos da IU podem ser divididos em conservador e cirúrgico. A Organização Mundial da Saúde recomenda que a IU seja tratada primeiramente através da fisioterapia e apenas após três meses de intervenção fisioterapêutica é que será avaliada a necessidade de um tratamento cirúrgico ou farmacológico ${ }^{2}$. Rodrigues et al. $^{8}$, observando tratamentos cirúrgicos, encontraram taxas de recorrência que variam de 5 a $20 \%$ nas abordagens por via abdominal e 10 a $64 \%$ quando se utiliza via vaginal.

A fisioterapia, por meio de exercícios pélvicos com ou sem biofeedback, estimulação elétrica e cones vaginais promove melhora da função neuromuscular do assoalho pélvico ${ }^{6}$. O procedimento consiste em educar a paciente a utilizar a musculatura estriada do assoalho pélvico adjacente à uretra para melhorar o controle da perda urinária ${ }^{2}$.

O Comitê de Padronização da Sociedade Internacional de Continência sugere cinco maneiras de observar os efeitos da intervenção terapêutica: sintomas da mulher, quantificação dos sintomas, observação clínica, qualidade de vida e medidas sócioeconômicas ${ }^{9}$.

A IU provoca alterações graves na qualidade de vida das pacientes, tornando-se estressante e debilitante, além de gerar alta morbidade por afetar o nível psicológico, ocupacional, doméstico, físico e sexual dos indivíduos por ela acometidos ${ }^{10}$.

Este trabalho teve como objetivos analisar a influência do tipo de parto em mulheres com IU na força muscular do assoalho pélvico antes e depois de uma intervenção fisioterapêutica e a relação dos tipos de parto com os tipos de IU.

\section{MATERIAIS E MÉTODOS}

Trata-se de um ensaio clínico, de braço único, não controlado, realizado no Hospital São Lucas (HSL) da Pontifícia Universidade Católica do Rio Grande do Sul (PUCRS), nos anos de 2006 a 2008, aprovado pelo Comitê de Ética em Pesquisa (CEP) da PUCRS (CEP 06/03194). Os princípios éticos foram respeitados de acordo com o estabelecido na resolução 196/96 do 
CNS-MS. As pacientes leram e assinaram o Termo de Consentimento Livre e Informado.

Foram incluídas 46 mulheres, com diagnóstico médico de IU (IUE, IUU e IUM), encaminhadas do ambulatório de Uroginecologia do HSL da PUCRS, para atendimento fisioterapêutico. As pacientes, antes do encaminhamento, foram orientadas a realizar exercícios domiciliares de reforço da musculatura pélvica diariamente por três meses sem obter resultados favoráveis.

Foram excluídas as pacientes que realizavam qualquer tipo de tratamento fisioterapêutico adicional, ou que iniciaram qualquer tipo de atividade física estruturada e planejada em adição àquelas previstas no protocolo do presente estudo ou que tinham se submetido à intervenção cirúrgica para correção de IU ou que apresentassem doenças como pneumopatias ou cardiopatias graves, doenças neurológicas ou oncológicas.

Todas as avaliações e intervenções foram realizadas no Ambulatório do Serviço de Fisioterapia do HSLPUCRS. Todos os procedimentos da avaliação inicial e da final foram realizados pelo mesmo examinador.

O estudo foi dividido em três fases:

1- Avaliação inicial: anamnese e medições prétratamento (perineometria e avaliação funcional do assoalho pélvico) foram realizadas no primeiro encontro.

2- Intervenção: foram realizadas até 15 sessões semanais (uma sessão/ semana) utilizando eletroestimulação transvaginal e exercícios perineais.

3- Avaliação final: assim que a paciente relatasse continência ou ao final das 15 sessões, foram realizadas as medições pós-tratamento (perineometria e avaliação funcional do assoalho pélvico).

A avaliação inicial e a final foram realizadas antes da primeira e da última sessão de tratamento. Na última sessão era pedido a todas as mulheres que relatassem como se consideravam ao final do tratamento, dentro de quatro opções: (1) continentes, (2) satisfeitas com o resultado do tratamento, (3) insatisfeitas ou (4) sem melhora. Assim, o relato das participantes foi adotado como forma de avaliação da perda urinária, seguindo as recomendações de Comitê de Padronização da Sociedade Internacional de Continência ${ }^{9}$.
$\mathrm{Na}$ anamnese foram coletados dados referentes a: idade, escolaridade, estado civil, tipo de IU, tempo da incontinência, situação(ões) de perda urinária, quantidade de urina perdida, número de gestações, tipo de parto (normal, cesárea, normal + cesárea), tempo decorrido desde o último parto, episiotomia, menopausa, obesidade, presença de prolapsos geniturinários. A quantidade de urina perdida foi descrita pela entrevistada como perda em gotas, jatos ou micção completa.

A avaliação funcional do assoalho pélvico foi realizada através da perineometria e da palpação bidigital, ambas com a mulher em posição de litotomia. Para a perineometria foi utilizado um perineômetro digital da marca Kroman, que detecta o aumento de pressão provocada pela contração do músculo elevador do ânus (MEA) através de um eletrodo transvaginal. A perineometria foi realizada três vezes e o maior valor em escala Sauers foi usado como referência. Posteriormente os valores obtidos foram convertidos em $\mathrm{cmH}_{2} \mathrm{O}$, considerando para conversão que 28 unidades Sauers equivalem a uma libra, que por sua vez corresponde a $0,49 \mathrm{cmH}_{2} \mathrm{O}$. A palpação bidigital foi graduada com base na escala de Ortiz ${ }^{11}$, que classifica o grau de contração entre 0 (sem função perineal objetiva, nem mesmo à palpação) e 5 (função perineal objetiva e com resistência opositora por mais de cinco segundos à palpação).

A intervenção fisioterapêutica envolveu eletroestimulação e cinesioterapia pélvica. A cinesioterapia consistiu de exercícios de fortalecimento do assoalho pélvico com auxílio de faixa elástica e bola. Os exercícios realizados foram de contração de abdutores e adutores de coxa com contração de períneo em decúbito dorsal e posição sentada e ponte pélvica com contração de períneo em decúbito dorsal. Foram realizadas duas séries de dez repetições, uma de contração lenta sustentada por cinco segundos e a outra de contração rápida para cada exercício descrito anteriormente.

A eletroestimulação foi realizada por um tempo de 10 minutos com intensidade conforme a tolerabilidade de cada paciente. Foi utilizado o aparelho Dualpex Uro da marca Quark, fabricado em Piracicaba - São Paulo, que 
tem eletrodo perineal transvaginal, o qual foi introduzido no canal vaginal das participantes para a aplicação da corrente. Os parâmetros usados para a eletroestimulação foram: frequência de $50 \mathrm{~Hz}$ para mulheres com IUE, $10 \mathrm{~Hz}$ para aquelas com IUU e associação das duas frequências em sessões alternadas para aquelas com IUM.

Tanto o número máximo de sessões utilizado nessa pesquisa (15 sessões) quanto a frequência das sessões (uma por semana) foram definidos a partir da prática de rotina no serviço de Fisioterapia do Ambulatório do HSLPUCRS. Se a paciente referisse continência ou satisfação antes das 15 sessões, o tratamento era dado por encerrado e a paciente reavaliada conforme protocolo da pesquisa. Porém, se após as 15 sessões a paciente não referisse continência ou satisfação com o tratamento, a mesma era reencaminhada ao Ambulatório de Uroginecologia do HSL - PUCRS para seguir com tratamento fora da pesquisa.

Foi feita a inserção dupla dos dados coletados para se eliminar erros de digitação, os quais foram posteriormente analisados através do software SPSS 13.0 (Statistical Package to Social Sciences for Windows) onde, para critérios de decisão foi adotado o nível de significância $(\alpha)$ de $5 \%$. A análise dos dados teve como abordagem inicial a estatística descritiva com a distribuição de frequências simples e relativa, bem como, as medidas de tendência central (média) e de variabilidade (desvio padrão). Ainda nesta primeira etapa foi investigada a distribuição dos dados através do teste de Kolmogorov-Smirnov. Na análise bivariada, que envolveu a comparação de médias em relação aos tipos de partos, foi utilizado o teste de Análise de variância (one way), ou o teste não-paramétrico equivalente, Kruskal-Wallys. Ainda na análise bivariada, na comparação das variáveis qualitativas em relação aos tipos de partos foi implementado o teste qui-quadrado de homogeneidade.

\section{RESULTADOS}

O estudo contou com 46 mulheres com média de idade de 53,6 $\pm 10,5$ anos (35 a 78 anos) das quais $60,9 \%$ apresentaram ensino fundamental completo ou incompleto e $67,4 \%$ informaram ser casadas. Agrupadas de acordo com o tipo de parto, as mulheres não diferiram estatisticamente em termos de idade (tabela 1).

A maior parte da amostra fez parto normal $(67,4 \%)$, 19,6\% fez parto normal e também cesárea e a minoria fez apenas cesárea (13\%). Essas mulheres tiveram analisados alguns fatores de risco para IU relativos ao tipo de parto (presença de prolapso, histerectomia, número de gestações e tempo de IU; tabela 1) e não diferiram significantemente, nem em relação à presença de fatores de risco e nem aos dados sóciodemográficos.

Quanto ao número de sessões de tratamento, a média foi de 12,91 $\pm 2,79$ sessões (5 a 15 sessões), sendo que $50 \%$ das pacientes realizaram entre 11 e 15 sessões. Não foi detectada diferença significativa entre o número de sessões e os tipos de parto, nem entre o número de sessões feitas e os tipos de IU.

Em relação ao tipo de incontinência, 50,0\% das pacientes investigadas apresentaram IUM, 39,1\% apresentaram IUE e $10,9 \%$ IUU. Analisando a relação entre os tipos de parto e os tipos de incontinência (tabela 1) observou-se que $54,8 \%$ das mulheres que realizaram parto normal apresentaram IUM, 83,3\% das mulheres que realizou cesárea apresentaram IUE e as mulheres que realizaram os dois tipos de parto, $55,6 \%$ apresentaram IUM e 44,4\% IUE. Apesar do teste Quiquadrado não apresentar poder de decisão e o número de mulheres que realizaram somente cesárea serem pequenos $(n=6)$, os dados sugerem que as pacientes que realizaram parto cesárea tendem a estar associadas à IUE, enquanto que aquelas que realizaram parto normal e normal e cesárea apresentaram uma maior inclinação às incontinências de esforço e mista.

Tomando como base a análise bivariada, não foram encontradas diferenças significativas na comparação do tipo de IU nem em relação ao tempo do último parto e nem à idade. Comparando a episiotomia em relação ao tipo de incontinência, o teste Qui-quadrado de homogeneidade não apontou associação estatística significativa entre as duas variáveis citadas $(p=0,395)$, indicando que a presença desse fator independe do tipo de incontinência.

Quanto ao relato das mulheres em relação à sua situação final em termos de continência urinária 
TABELA 1 - Características da amostra de acordo com o tipo de parto.

\begin{tabular}{|c|c|c|c|c|c|}
\hline \multirow[b]{2}{*}{ Variáveis } & \multicolumn{4}{|c|}{ Tipo de Parto } & \multirow[b]{2}{*}{$\mathbf{P}$} \\
\hline & Total & Normal & Cesárea & $\mathrm{N}+\mathrm{C}$ & \\
\hline & $(n=46)$ & $(n=31)$ & $(n=6)$ & $(n=9)$ & \\
\hline \multicolumn{6}{|l|}{ Idade (anos) } \\
\hline Média $( \pm D P)$ & $53,8(10,8)$ & $50,9(8,2)$ & $59,3(12,1)$ & $54,8(12,1)$ & \multirow[t]{2}{*}{$0,221 \Theta$} \\
\hline Min - Máx & $35-78$ & $37-67$ & $42-77$ & $35-78$ & \\
\hline \multicolumn{6}{|l|}{ Tipo de IU* } \\
\hline Esforço & $18(39,1)$ & $9(29,0)$ & $5(83,3)$ & $4(44,4)$ & \multirow{3}{*}{$0,098 \phi$} \\
\hline Urgência & $5(10,9)$ & $5(16,1)$ & $0(0,0)$ & $0(0,0)$ & \\
\hline Mista & $23(50,0)$ & $17(54,8)$ & $1(16,7)$ & $5(55,6)$ & \\
\hline \multicolumn{6}{|c|}{ Tempo de IU (anos) } \\
\hline Média ( $(\mathrm{DP})$ & $7,5(5,9)$ & $8,1(6,7)$ & $6,2(1,6)$ & $6,3(4,4)$ & 0,861 • \\
\hline \multicolumn{6}{|l|}{ № Gestações* } \\
\hline Uma & $6(13,0)$ & $5(16,1)$ & $1(16,7)$ & $0(0,0)$ & \multirow{4}{*}{0,390} \\
\hline Duas & $12(26,1)$ & $9(29,0)$ & $2(33,3)$ & $1(11,1)$ & \\
\hline Três & $13(28,3)$ & $6(19,4)$ & $2(33,3)$ & $5(55,5)$ & \\
\hline Quatro ou mais & $15(32,6)$ & $11(35,5)$ & $1(16,7)$ & $3(33,3)$ & \\
\hline \multicolumn{6}{|c|}{ Tempo desde o último parto (anos) } \\
\hline Média $( \pm \mathrm{DP})$ & $22,0(12,8)$ & $19,7(11,1)$ & $17,2(16,7)$ & $24,9(13,3)$ & 0,427 • \\
\hline \multicolumn{6}{|l|}{ Prolapso* } \\
\hline $\operatorname{Sim}$ & $35(76,1)$ & $22(71,0)$ & $6(100,0)$ & $7(77,8)$ & \multirow{2}{*}{$0,309 \S$} \\
\hline Não & $11(23,9)$ & $9(29,0)$ & $0(0,0)$ & $2(22,2)$ & \\
\hline \multicolumn{6}{|l|}{ Histerectomia* } \\
\hline $\operatorname{Sim}$ & $3(6,8)$ & $2(6,7)$ & $1(16,7)$ & $0(0,0)$ & \multirow{2}{*}{$0,472 \phi$} \\
\hline Não & $41(93,2)$ & $28(93,3)$ & $5(83,3)$ & $8(100,0)$ & \\
\hline \multicolumn{6}{|l|}{ Situação final $¥^{*}$} \\
\hline Continente & $20(43,5)$ & $13(41,9)$ & $2(33,3)$ & $5(55,6)$ & \multirow{4}{*}{$0,741 \phi$} \\
\hline Satisfeita & $20(43,5)$ & $13(41,9)$ & $3(50,0)$ & $4(44,4)$ & \\
\hline Insatisfeita & $3(6,5)$ & $2(6,5)$ & $1(16,7)$ & $0(0,0)$ & \\
\hline Sem melhora & $3(6,5)$ & $3(9,7)$ & $0(0,0)$ & $0(0,0)$ & \\
\hline
\end{tabular}

$\mathrm{N}+\mathrm{C}$ : parto normal e cesárea; p: probabilidade; DP: desvio padrão; $\theta$ : ANOVA; IU: incontinência urinária; *valores apresentados na forma de $\mathrm{n}(\%) ; \phi$ : teste qui-quadrado de Pearson; §: teste qui-quadrado de Pearson; $\bullet:$ teste de KruskalWallys; ¥: como as mulheres se consideravam ao final do tratamento fisioterapêutico.

(tabela 1), após o tratamento $87,0 \%$ do total da amostra informaram estar continentes ou satisfeitas e apenas $6,5 \%$ informaram não terem apresentado melhora. $\mathrm{Na}$ comparação da situação final das mulheres levando em consideração os tipos de partos (tabela 1), 83,8\% daquelas que realizaram parto normal relataram estar continentes e satisfeitas, percentual semelhante àquele encontrado entre as pacientes que realizaram cesárea $(83,3 \%)$. No grupo de pacientes que realizou os dois tipos de partos (normal e cesárea) a situação de continência e satisfação foi de 100,0\%.

Na comparação do total da amostra entre as medidas do perineômetro antes e depois do tratamento (tabela 2), o teste t-Student não apontou diferença estatística significativa $(p=0,185)$. Esse mesmo resultado também foi encontrado, quando os dados foram analisados em termos do tipo de IU, onde não foram detectadas diferenças estatisticamente significativas $(p>0,05)$ nas comparações entre as avaliações antes e depois para o perineômetro.

$\mathrm{Na}$ tabela 2 também pode ser observado que, na comparação dos valores do perineômetro antes do tratamento entre os diferentes tipos de parto, a análise de variância (one-way) apontou diferença estatística significativa $(p=0,011)$, indicando que as pacientes que realizaram parto normal apresentaram força muscular do assoalho pélvico significativamente menor que as pacientes que realizaram cesárea, que por sua vez, 
TABELA 2 - Comparação dos achados do perineômetro (média \pm desvio padrão) antes e depois da intervenção fisioterapêutica, segundo o tipo de parto.

\begin{tabular}{lccc}
\hline & \multicolumn{3}{c}{ Perineômetro } \\
Tipo de parto & Antes $(\mathbf{n}=46)$ & Depois $(\mathbf{n}=46)$ & $\mathbf{p}$ \\
\hline Normal & $34,8(22,1)$ & $40,9(24,7)$ & $0,031 \Theta$ \\
Cesárea & $48,7(30,6)$ & $50,0(23,4)$ & $0,840 \Theta$ \\
Normal e & $63,1(26,7)$ & $60,3(30,6)$ & $0,804 \Theta$ \\
Cesárea & & & \\
$\mathbf{p}$ & 0,011 & 0,145 & \\
\hline
\end{tabular}

p: probabilidade; $\bullet$ : teste t de Student; $\bullet$ : ANOVA.

apresentaram uma força muscular significativamente menor que a força apresentada pelo grupo de pacientes que realizou os dois tipos de parto. Já na comparação entre as medidas do perineômetro em cada tipo de parto feitas antes e depois do tratamento fisioterapêutico foi encontrada diferença significativa apenas entre as mulheres que realizaram parto normal $(p=0,031$; teste tStudent para amostras pareadas), as quais apresentaram um aumento médio da força de 18,9\%.

Foi detectado ganho de força significativo após o tratamento fisioterapêutico ( $p<0,01$; teste Wilcoxon) nas medidas do teste bidigital dos MAP para o total da amostra. Nas comparações do MAP (antes e depois) entre os diferentes tipos de parto (tabela 3), foi observado que as mulheres que realizaram parto normal apresentaram ganho de força significativo $(p=0,018)$, enquanto as pacientes que realizaram cesárea e as que realizaram os dois tipos de parto não apresentaram ganho estatisticamente significativo ( $p>0,05$; teste Wilcoxon).

Ainda apresentando os resultados da tabela 3, tanto na comparação das medidas dos MAP antes do tratamento em relação aos tipos de parto, quanto na comparação após, o teste de Kruskal-Wallys não apontou diferença estatística significativa $(p>0,05)$, indicando que, independente do tipo de parto as medidas observadas se mostraram semelhantes.

\section{DISCUSSÃO}

O parto normal foi o mais prevalente, o que era esperado, considerando que o total da amostra foi composto por usuárias do Sistema Único de Saúde. E a forma mais prevalente de IU foi a mista, seguida pela
TABELA 3 - Comparação do grau de força muscular do assoalho pélvico (média \pm desvio padrão) medido por meio do teste bidigital, antes e depois da intervenção fisioterapêutica, segundo o tipo de parto.

\begin{tabular}{lccc}
\hline & \multicolumn{3}{c}{ Força Muscular do Assoalho Pélvico } \\
Tipo de Parto & Antes $(\mathbf{n}=46)$ & Depois $(\mathbf{n = 4 6 )}$ & $\mathbf{p}$ \\
\hline Normal & $3,8(1,0)$ & $4,3(0,9)$ & $0,018 \Theta$ \\
Cesárea & $3,7(1,5)$ & $4,7(0,8)$ & $0,063 \Theta$ \\
$\begin{array}{l}\text { Normal e } \\
\text { Cesárea }\end{array}$ & $4,2(0,8)$ & $4,7(0,5)$ & $0,102 \Theta$ \\
$\quad$ p & $0,507 \bullet$ & 0,282 & \\
\hline
\end{tabular}

p: probabilidade; ө: teste de Wilcoxon; $\bullet$ : teste de Kruskal-Wallys.

IUE, confirmando os achados de Figueiredo et al. ${ }^{12}$ em trabalho realizado com mulheres portadoras de IU com características semelhantes àquelas que participaram do presente estudo.

Analisando a relação entre os tipos de parto e os tipos de incontinência (tabela 1), foi observado que a maioria das mulheres que realizaram parto normal apresentou IUM, enquanto que a maioria das mulheres que realizaram cesáreas apresentaram IUE e as mulheres que realizaram os dois tipos de parto se dividiram entre IUM e IUE. Esses resultados diferem daqueles obtidos por Rortveit et al. ${ }^{13}$, que encontraram associação entre tipo de parto e IUE, enquanto um risco aumentado para IUE e IUM foi associado à gravidez.

As mensurações feitas com o perineômetro não apresentaram aumento significativo após a intervenção fisioterapêutica. Este achado pode estar relacionado com o grande número de mulheres que apresentaram prolapso (76,1\%), uma vez que o aumento da força muscular resultante do tratamento fisioterapêutico da IU pode melhorar o posicionamento das vísceras pélvicas, o que por sua vez, pode levar à diminuição da massa presente no interior do canal vaginal e, consequentemente, levar a uma diminuição no tamanho do prolapso. Isso pode levar a menores leituras registradas pelo perineômetro no período póstratamento $^{14}$.

Segundo Menta e Schirmer ${ }^{15}$, a musculatura pélvica compõe-se de fibras do tipo I (cerca de 70\%) e do tipo II $(30 \%)$, sendo as primeiras responsáveis pela manutenção do tônus muscular, pela capacidade de contração lenta e de suportar longos períodos de solicitação sem sofrer fadiga. As fibras do tipo II são 
fatigáveis, realizam as contrações rápidas em resposta a aumentos súbitos de pressão intra-abdominal, o que acontece em situações de tosse ou esforço repentino, porém não em trabalho de parto, uma vez que este constitui processo lento e gradual.

Em nosso estudo, nas mensurações feitas com o teste bidigital, constatamos que as pacientes que realizaram apenas parto normal obtiveram ganho de força muscular significativo MAP; as pacientes que realizaram parto cesárea obtiveram ganho de força muscular menor, enquanto que as que realizaram os dois tipos de parto não apresentaram aumento significativo. Na literatura pesquisada não foi encontrado resultado que confirmasse ou que contrapusesse esses achados, ou mesmo que apresentasse justificativa para esses diferentes tipos de resposta ao tratamento fisioterapêutico, que, em termos de cinesioterapia foi aplicado de forma uniforme a todas as participantes do estudo.

A única diferença na aplicação do tratamento fisioterapêutico se deu nos parâmetros da corrente de eletroestimulação para IUE (18/46 mulheres) e IUM (23/46), tipos de IU predominantes nos três tipos de parto. Conforme exposto na seção Métodos do presente artigo, da média de 13 sessões feitas pelas voluntárias com IUM, em pelo menos seis delas as mulheres receberam a eletroestimulação com os mesmo parâmetros de corrente usadas pelas mulheres com IUE. Ainda assim, não se consegue explicar as razões pelas quais as mulheres do presente estudo que fizeram parto normal apresentaram ganho significativo de força muscular do assoalho pélvico.

Para responder a essa questão teríamos que realizar uma análise multivariada. Entretanto, levando em consideração o tamanho da presente amostra, em particular o número de casos com IUU, não seria possível aplicar esse tipo de técnica. Considerando que foram necessários dois anos para atingir esse tamanho amostral e que, continuando as mesmas condições de encaminhamento, mesmo no dobro desse tempo teríamos ainda um número pequeno de mulheres com IUU ( $n \approx 12)$, é provável que essa resposta só seja plausível em um período de tempo muito mais longo do que foi possível no presente estudo.
Há controvérsia na literatura sobre a suposta proteção dada ao assoalho pélvico pelo parto cesárea, comparado com o normal. Mckinnie et al. ${ }^{16}$ não encontraram diferença no risco de IU e incontinência fecal entre mulheres que realizaram parto cesárea e mulheres que realizaram parto normal. Foldspang et al. ${ }^{17}$ concluem que, na verdade é todo o processo da gestação e não apenas o parto normal que está fortemente associado com a prevalência de IUE.

Adicionalmente, estudos mostram que as diferenças na força do assoalho pélvico entre mulheres que realizaram parto normal e as que realizaram cesárea não são significativas ${ }^{6,16}$. Barbosa et al. ${ }^{6}$ avaliaram a força muscular do assoalho pélvico em primíparas quatro a seis meses após o parto e encontraram diminuição da força tanto no parto normal como na cesárea, observando que o parto normal teve uma diminuição mais acentuada. Menta e Schirmer ${ }^{16}$ encontraram que as mulheres submetidas ao parto cesárea tiveram valores médios de pressão muscular perineal maiores do que aquelas com episiotomia, ruptura perineal e integridade perineal, porém, essa diferença não se mostrou significativa.

O número de sessões necessárias para a continência ou melhora variou bastante, entre apenas cinco e o máximo de 15 oferecidas. Mesmo o número máximo de sessões sendo pequeno e tendo baixos custos, $87 \%$ do total da amostra informaram estar continentes ou satisfeitas com 0 tratamento realizado, não necessitando de tratamento adicional e sem relatos de efeitos adversos. Esse alto grau de satisfação foi encontrado, também, quando os mesmos dados foram avaliados de acordo com o tipo de parto realizado, demonstrando que o tratamento foi bem sucedido, independente do tipo de parto ou mesmo do tipo de IU. Estes resultados relativos à satisfação das pacientes vem de encontro aos achados de Herrmann et al. ${ }^{18}$, que relataram cura ou melhora em $81,7 \%$ das mulheres com IUE após tratamento apenas com eletroestimulação intravaginal.

Os resultados apresentados refletem, portanto, o fato de que o tratamento fisioterapêutico, mesmo realizado apenas uma vez por semana, traz benefícios à saúde das pacientes, podendo levá-las à melhora ou até a cura 
da IU. Enquanto 0 teste bidigital demonstrou sensibilidade na detecção desse aumento de força muscular e continência obtidos, o perineômetro não. Esse último instrumento é caro e implica em gastos para sua utilização e manutenção, podendo, portanto, ser excluído da avaliação rotineira das portadoras de IU. Dado esse achado e o de que o teste bidigital pode ser utilizado para observar os efeitos da intervenção terapêutica, assim como o alto percentual de mulheres satisfeitas e/ou continentes após o tratamento utilizado no presente estudo, pode-se sugerir que intervenções fisioterapêuticas como a que foi utilizada possam ser adotadas como parte da rotina do cuidado à saúde da mulher na rede de saúde pública.

Concluímos que o tipo de parto parece não influenciar a ocorrência de IU, nem a força muscular do assoalho pélvico ou mesmo o sucesso da intervenção fisioterapêutica, uma vez que a maioria das pacientes relatou estar continente ou satisfeita após o tratamento.

\section{REFERÊNCIAS}

1. Souza ELBL. Fisioterapia aplicada à obstetrícia uroginecologia e aspectos de mastologia. $4^{\underline{a}}$ ed. Rio de Janeiro: Guanabara Koogan; 2007.

2. Parente LC, Henriques SHFC. Fisioterapia na incontinência urinária no pós-parto. Rev Fisiot Brasil 2007; 8(04):288-93.

3. Dumoulin C, Hay-Smith J. Pelvic floor muscle training versus no treatment, or inactive control treatments, for urinary incontinence in women. Cochrane Database of Systematic Reviews 2006; Issue 1. Art. No.: CD005654. DOI: $10.1002 / 14651858 . C D 005654$.

4. Abrams $P$, Cardoso L, Tall M, Grittitus $D$, Rosier $P$, Ulmsten $U$, Kerrebroeck $P$, Victor $A$, Wein $A$. The standardisation of terminology in lower urinary tract function: report from de standardisation sub-committee of the international continence society. Urology 2003; 61(01): 37-49.

5. Scarpa KP, Herrmann V, Palma PCR, Ricetto CLZ, Morais $\mathrm{S}$. Sintomas do trato urinário inferior três anos após o parto: estudo prospectivo. Rev Bras Ginecol Obstet. 2008; 30(7):355-9.

6. Barbosa AMP, Carvalho LR, Martins AMVC, Calderon IMP, Rudge MVC. Efeito da via de parto sobre a força muscular do assoalho pélvico. Rev Bras Ginecol Obstet. 2005; 27(11): 677-82.

7. Oliveira SMJV, Miquilini EC. Frequência e critérios para indicar a episiotomia. Rev Esc Enferm USP. 2005; 39(3): 288-95.

8. Rodrigues NNJ. Incontinência urinária de esforço na mulher. Rev Assoc Med Bras. 1990; 36:15-7.

9. Royal College of Obstetricians and Gynaecologists. Urinary incontinence: the management of urinary incontinence in women. London: RCOG Press; 2006. Disponível em: http://www.nice.org.uk/nicemedia/pdf/ CG40fullguideline.pdf

10. Tamanini JTN, D'Ancona CAL, Botega NJ, Rodrigues NNJ. Validação do "King's Health Questionnaire" para o português em mulheres com incontinência urinária. Rev Saúde Pública. 2003; 37(02):203-11.

11. Ortiz $O C$ et al. Valoración dinâmica de la disfunción perineal da classificación. Boletim de La Sociedad Latino Americana de Uroginecologia y Cirurgia Vaginal. 1994; 1(2):7-9.

12. Figueiredo EM, Lara JO, Cruz MC, Quintão DMG, Monteiro MVC. Perfil sócio demográfico e clínico de usuárias de serviço de fisioterapia uroginecológica da rede pública. Rev Bras Fisioter. 2008; 12(2):136-42.

13. Rortveit G, Daltveit AK, Hannestad YS, Hunskaar S. Urinary Incontinence after vaginal delivery or cesarean section. N Engl J Med. 2003; 348(10):900-7.

14. Neumann $P$, Morrison S. Physiotherapy for urinary incontinence. Aust Fam Physician. 2008; 37(3): 18-21.

15. Menta $S$, Schirmer J. Relação entre a pressão muscular perineal no puerpério e o tipo de parto. Rev Bras Ginecol Obstet. 2006; 28(9):523-9.

16. McKinnie V, Swift SE, Wang W, Woodman P, O'Boyle A, Kahn M, Valley M, Bland D, Schaffer J. The effect of pregnancy and mode of delivery on the prevalence of urinary and fecal incontinence. Am J Obstet Ginecol. 2005; 193:512-8.

17. Foldspang A, Mommsen S, Djurhws J. Prevalence urinary incontinence as a correlate of pregnancy, vaginal childbirth and obstetric techniques. Am J Public Health. 1999; 89(2): 209-12.

18. Herrmann V, Potrick BA, Palma PCR, Zanettini CL, Marques A, Netto NRJ. Eletroestimulação transvaginal do assoalho pélvico no tratamento da incontinência urinaria de esforço: avaliações clinica e ultra-sonográfica. Rev Assoc Med Bras. 2003; 49(4):401-5.

\section{Endereço para correspondência:}

Mara Regina Knorst

Av. Ipiranga 6681 - Prédio 12 - 8ํanda

Porto Alegre/RS - CEP 90619-900

Telefone: +55 5133203646

E-mail: mknorst@pucrs.br 